\title{
Rough Multilinear Fractional Integrals on Weighted Morrey Spaces
}

\author{
Xiao $\mathrm{Li}^{1}$ and Runqing $\mathrm{Cui}^{2}$ \\ ${ }^{1}$ Department of Foundation, Zhejiang University of Water Resources and Electric Power, Hangzhou 310018, China \\ ${ }^{2}$ College of Mathematics and Informatics, Henan Polytechnic University, Jiaozuo 454003, China
}

Correspondence should be addressed to Xiao Li; hzlixiao@yeah.net

Received 23 May 2015; Revised 15 July 2015; Accepted 21 July 2015

Academic Editor: Henryk Hudzik

Copyright (c) $2015 \mathrm{X}$. Li and R. Cui. This is an open access article distributed under the Creative Commons Attribution License, which permits unrestricted use, distribution, and reproduction in any medium, provided the original work is properly cited.

It is showed that a class of multilinear fractional operators with rough kernels, which are similar to the higher-order commutators for the rough fractional integrals, are bounded on the weighted Morrey spaces.

\section{Introduction and Results}

Suppose that $\mathbb{S}^{n-1}$ denotes the unit sphere of $\mathbb{R}^{n}(n \geq$ 2) equipped with the usual Lebesgue measure, $\Omega \in$ $L^{s}\left(\mathbb{S}^{n-1}\right)(s>1)$ is homogeneous of degree zero, and $A_{j}, j=$ $1, \ldots, k$, are functions defined on $\mathbb{R}^{n}$. Consider the following multilinear fractional integral with rough kernel defined by

$$
\begin{aligned}
& T_{\Omega, \alpha}^{A_{1}, \ldots, A_{k}}(f)(x) \\
& \quad=\int_{\mathbb{R}^{n}} \frac{\Omega(x-y)}{|x-y|^{n-\alpha+N}} \prod_{j=1}^{k} R_{m_{j}}\left(A_{j} ; x, y\right) f(y) d y,
\end{aligned}
$$

where $0<\alpha<n, N=\sum_{j=1}^{k}\left(m_{j}-1\right)$, and

$$
R_{m_{j}}\left(A_{j} ; x, y\right)=A_{j}(x)-\sum_{|\gamma|<m_{j}} \frac{1}{\gamma !} D^{\gamma} A_{j}(y)(x-y)^{\gamma} .
$$

When $k=1, A_{1}=A$, and $m_{1}=1$, then $T_{\Omega, \alpha}^{A}$ is just the commutator of the rough fractional integral $T_{\Omega, \alpha}$ with the function $A$ :

$$
\begin{aligned}
& {\left[A, T_{\Omega, \alpha}\right] f(x)} \\
& \quad=\int_{\mathbb{R}^{n}} \frac{\Omega(x-y)}{|x-y|^{n-\alpha}}(A(x)-A(y)) f(y) d y .
\end{aligned}
$$

If $m_{1} \geq 2$, then $T_{\Omega, \alpha}^{A}$ is nontrivial generalization of the above commutator. The weighted $\left(L^{p}, L^{q}\right)$-boundedness of the operator $T_{\Omega, \alpha}^{A}$ was given by Wu and Yang in [1].

When $k>1, A_{j}=A$ for $j=1, \ldots, k$, and $m_{j}=1$, then $T_{\Omega, \alpha}^{A_{1}, \ldots, A_{k}}$ is the higher-order commutator of the fractional integral $T_{\Omega, \alpha}$ with the function $A$ :

$$
T_{\Omega, \alpha}^{A, k} f(x)=\int_{\mathbb{R}^{n}} \frac{\Omega(x-y)}{|x-y|^{n-\alpha}}(A(x)-A(y))^{k} f(y) d y .
$$

The weighted $\left(L^{p}, L^{q}\right)$-boundedness of $T_{\Omega, \alpha}^{A, k}$ was given by Ding and Lu in [2].

Ding and Lu in [3] proved the following result.

Theorem 1. Let $0<\alpha<n, 1<p<n / \alpha$, let $1 / q=$ $1 / p-\alpha / n$, and let $\Omega$ be homogeneous of degree zero with $\Omega \in L^{s}\left(\mathbb{S}^{n-1}\right), s>1$. Moreover, for $1 \leq j \leq k,|\gamma|=m_{j}-1$, $m_{j} \geq 2$, and $D^{\gamma} A_{j} \in \operatorname{BMO}\left(\mathbb{R}^{n}\right)$. If $\omega^{s^{\prime}} \in A\left(p / s^{\prime}, q / s^{\prime}\right)$, then there exists a constant $C$, independent of $A_{j}(1 \leq j \leq k)$ and $f$, such that

$$
\begin{aligned}
& \left\|T_{\Omega, \alpha}^{A_{1}, \ldots, A_{k}} f\right\|_{L^{q}\left(\omega^{q}, \mathbb{R}^{n}\right)} \\
& \quad \leq C\left(\prod_{j=1}^{k} \sum_{|\gamma|=m_{j}-1}\left\|D^{\gamma} A_{j}\right\|_{*}\right)\|f\|_{L^{p}\left(\omega^{p}, \mathbb{R}^{n}\right)} .
\end{aligned}
$$


Here and in the sequel, $p^{\prime}$ always denotes the conjugate index of any $p>1$; that is, $1 / p+1 / p^{\prime}=1$, and $C$ stands for a constant which is independent of the main parameters, but it may vary from line to line.

The purpose of this paper is to discuss the boundedness properties of the rough fractional multilinear integral operators $T_{\Omega, \alpha}^{A_{1}, \ldots, A_{k}}$ on appropriate weighted Morrey spaces.

The classical Morrey spaces were introduced by Morrey [4] in 1938 and have been studied intensively by various authors and together with weighted Lebesgue spaces play an important role in the theory of partial differential equations [5-7]. by

Let $1 \leq p<\infty$ and $0 \leq \lambda \leq n$; Morrey spaces are defined

$$
L^{p, \lambda}\left(\mathbb{R}^{n}\right)=\left\{f \in L_{\mathrm{loc}}^{p}\left(\mathbb{R}^{n}\right):\|f\|_{L^{p, \lambda}\left(\mathbb{R}^{n}\right)}<\infty\right\},
$$

where

$$
\|f\|_{L^{p, \lambda}\left(\mathbb{R}^{n}\right)}=\sup _{x \in \mathbb{R}^{n}, r>0} r^{-\lambda / p}\|f\|_{L^{p}(B(x, r))} .
$$

Note that $L^{p, 0}\left(\mathbb{R}^{n}\right)=L^{p}\left(\mathbb{R}^{n}\right)$ and $L^{p, n}\left(\mathbb{R}^{n}\right)=L^{\infty}\left(\mathbb{R}^{n}\right)$. If $\lambda<0$ or $\lambda>n$, then $L^{p, \lambda}\left(\mathbb{R}^{n}\right)=\Theta$, where $\Theta$ is the set of all functions equivalent to 0 on $\mathbb{R}^{n}$.

Let $\Phi(r), r>0$, be a growth function, that is, a positive increasing function in $(0, \infty)$, which satisfies doubling condition

$$
\Phi(2 r) \leq D \Phi(r), \quad \forall r>0,
$$

where $D=D(\Phi) \geq 1$ is a doubling constant independent of $r$. Mizuhara in [8] gave generalization Morrey spaces $L^{p, \Phi}\left(\mathbb{R}^{n}\right)$ considering $\Phi(r)$ instead of $r^{\lambda}$ in (7).

Komori and Shirai [9] introduced a version of the weighted Morrey space $L^{p, \kappa}\left(\omega, \mathbb{R}^{n}\right)$, which is a natural generalization of the weighted Lebesgue spaces $L^{p}\left(\omega, \mathbb{R}^{n}\right)$.

Let $1 \leq p<\infty, 0<\kappa<1$, and $\omega$ a weight function. The spaces $L^{p, \kappa}\left(\omega, \mathbb{R}^{n}\right)$ are defined by

$$
L^{p, \kappa}\left(\omega, \mathbb{R}^{n}\right)=\left\{f \in L_{\mathrm{loc}}^{p}(\omega):\|f\|_{L^{p, \kappa}\left(\omega, \mathbb{R}^{n}\right)}<\infty\right\},
$$

where

$$
\begin{gathered}
\|f\|_{L^{p, \kappa}\left(\omega, \mathbb{R}^{n}\right)}=\sup _{x \in \mathbb{R}^{n}, r>0}\left(\frac{1}{\omega(B(x, r))^{\kappa}}\right. \\
\left.\cdot \int_{B(x, r)}|f(y)|^{p} \omega(y) d y\right)^{1 / p} .
\end{gathered}
$$

In order to deal with the fractional order case, we need to consider the weighted Morrey spaces with two weight functions; they were also introduced by Komori and Shirai in [9].

Let $1 \leq p<\infty, 0<\kappa<1$. For two weight functions $u$ and $v$, the spaces $L^{p, \kappa}(u, v)\left(\mathbb{R}^{n}\right)$ are defined by

$$
L^{p, \kappa}(u, v)\left(\mathbb{R}^{n}\right)=\left\{f \in L_{\mathrm{loc}}^{p}(u):\|f\|_{L^{p, \kappa}(u, v)}<\infty\right\},
$$

where

$$
\begin{aligned}
& \|f\|_{L^{p, \kappa}(u, v)\left(\mathbb{R}^{n}\right)} \\
& =\sup _{x \in \mathbb{R}^{n}, r>0}\left(\frac{1}{v(B(x, r))^{\kappa}} \int_{B(x, r)}|f(y)|^{p} u(y) d y\right)^{1 / p} .
\end{aligned}
$$

Let $1 \leq p<\infty, \varphi$ a positive measurable function on $\mathbb{R}^{n} \times(0, \infty)$, and $\omega$ a nonnegative measurable function on $\mathbb{R}^{n}$. We denote by $M_{\varphi}^{p}\left(\omega, \mathbb{R}^{n}\right)$ other weighted Morrey spaces, the spaces of all functions $f \in L_{\mathrm{loc}}^{p}(\omega)$ with finite norm

$$
\begin{aligned}
& \|f\|_{M_{\varphi}^{p}\left(\omega, \mathbb{R}^{n}\right)} \\
& \quad=\sup _{x \in \mathbb{R}^{n}, r>0} \frac{1}{\varphi(x, r)}\left(\frac{1}{w(B(x, r))}\|f\|_{L^{p}(\omega, B(x, r))}^{p}\right)^{1 / p} \\
& \quad<\infty,
\end{aligned}
$$

where

$$
\|f\|_{L^{p}(\omega, B(x, r))}=\int_{B(x, r)}|f(y)|^{p} \omega(y) d y .
$$

Remark 2. (1) If $\omega=1$ and $\varphi(x, r)=r^{(\lambda-n) / p}$ with $0 \leq \lambda \leq n$, then $M_{\varphi}^{p}\left(\omega, \mathbb{R}^{n}\right)=L^{p, \lambda}\left(\mathbb{R}^{n}\right)$.

(2) If $\omega=1, \varphi(x, r)^{p}=\Phi(r) r^{-n}$, then $M_{\varphi}^{p}\left(\omega, \mathbb{R}^{n}\right)=$ $L^{p, \Phi}\left(\mathbb{R}^{n}\right)$.

(3) If $\varphi(x, r)=\omega(B(x, r))^{(\kappa-1) / p}$, then $M_{\varphi}^{p}\left(\omega, \mathbb{R}^{n}\right)=$ $L^{p, \kappa}\left(\omega, \mathbb{R}^{n}\right)$.

(4) If $\varphi(x, r)=\nu(B(x, r))^{\kappa / p} \omega(B(x, r))^{-1 / p}$, then $M_{\varphi}^{p}\left(\omega, \mathbb{R}^{n}\right)=L^{p, \kappa}(\nu, \omega)\left(\mathbb{R}^{n}\right)$.

Our main results can be formulated as follows.

Theorem 3. Let $0<\alpha<n, 1<p<n / \alpha$, let $1 / q=1 / p-\alpha / n$, and let $\Omega$ be homogeneous of degree zero with $\Omega \in L^{s}\left(\mathbb{S}^{n-1}\right)$, $s>1$. Suppose $\omega^{s^{\prime}} \in A\left(p / s^{\prime}, q / s^{\prime}\right)$ and $\left(\varphi_{1}, \varphi_{2}\right)$ satisfies the condition

$$
\begin{aligned}
& \int_{s}^{\infty}\left(1+\ln \frac{r}{s}\right)^{k} \\
& \cdot \frac{\operatorname{ess~inf}_{r<t<\infty} \varphi_{1}(x, t)\left(\omega^{p}(B(x, t))\right)^{1 / p}}{\left(\omega^{q}(B(x, r))\right)^{1 / q}} \frac{1}{r} d r \\
& \quad \leq C_{0} \varphi_{2}(x, s),
\end{aligned}
$$

where $C_{0}$ does not depend on $x$ and $r$. If $A_{j}$ have derivatives of order $m_{j}-1$ in $B M O\left(\mathbb{R}^{n}\right), j=1, \ldots, k$, then there is a constant $C>0$, independent of $A_{j}(1 \leq j \leq k)$ and $f$, such that

$$
\begin{aligned}
& \left\|T_{\Omega, \alpha}^{A_{1}, \ldots, A_{k}} f\right\|_{M_{\varphi_{2}}^{q}\left(\omega^{q}, \mathbb{R}^{n}\right)} \\
& \quad \leq C\left(\prod_{j=1}^{k} \sum_{|\gamma|=m_{j}-1}\left\|D^{\gamma} A_{j}\right\|_{*}\right)\|f\|_{M_{\varphi_{1}}^{p}\left(\omega^{p}, \mathbb{R}^{n}\right)} .
\end{aligned}
$$


Remark 4. Let $\varphi_{1}(x, t)=\left(\Phi(t) t^{-n}\right)^{1 / p}$, let $\varphi_{2}(x, t)=$ $(\Phi(t))^{1 / p} t^{n / q}$, and let $\omega=1$. If $1 \leq D(\Phi) \leq 2^{n}$, it is easy to prove that $\left(\varphi_{1}, \varphi_{2}\right)$ satisfies condition (15).

Remark 5. Let $\varphi_{1}(x, t)=\left(\omega^{q}(B(x, t))\right)^{\kappa / p}\left(\omega^{p}(B(x, t))\right)^{-1 / p}$, $\varphi_{2}(x, t)=\left(\omega^{q}(B(x, t))\right)^{\kappa / p-1 / q}, 0<\kappa<p / q$, and $w^{q} \epsilon$ $A_{\infty}\left(\mathbb{R}^{n}\right)$. By [10], we know that $\left(\varphi_{1}, \varphi_{2}\right)$ satisfies condition (15).

Corollary 6. Let $0<\alpha<n, 1<p<n / \alpha$, let $1 / q=$ $1 / p-\alpha / n$, and let $\Omega$ be homogeneous of degree zero with $\Omega \in L^{s}\left(\mathbb{S}^{n-1}\right), s>1$. Suppose $1 \leq D(\Phi) \leq 2^{n}$, and $A_{j}$ have derivatives of order $m_{j}-1$ in $B M O\left(\mathbb{R}^{n}\right), j=1, \ldots, k$; then $T_{\Omega, \alpha}^{A_{1}, \ldots, A_{k}}$ is bounded from $L^{p, \Phi}\left(\mathbb{R}^{n}\right)$ to $L^{q, \Phi^{q / p}}\left(\mathbb{R}^{n}\right)$ and

$$
\begin{aligned}
& \left\|T_{\Omega, \alpha}^{A_{1}, \ldots, A_{k}} f\right\|_{L^{q, \Phi q / p}\left(\mathbb{R}^{n}\right)} \\
& \quad \leq C\left(\prod_{j=1}^{k} \sum_{|\gamma|=m_{j}-1}\left\|D^{\gamma} A_{j}\right\|_{*}\right)\|f\|_{L^{p, \Phi}\left(\mathbb{R}^{n}\right)} .
\end{aligned}
$$

Remark 7. In Corollary 6, let $k=1$ and $m_{1}=1$; then we obtain the main result in [11].

Corollary 8. Let $0<\alpha<n, 1<p<n / \alpha$, let $1 / q=1 / p-\alpha / n$, and let $\Omega$ be homogeneous of degree zero with $\Omega \in L^{s}\left(\mathbb{S}^{n-1}\right)$, $s>1$. Suppose $0<\kappa<p / q, \omega^{s^{\prime}} \in A\left(p / s^{\prime}, q / s^{\prime}\right)$, and $A_{j}$ have derivatives of order $m_{j}-1$ in $B M O\left(\mathbb{R}^{n}\right), j=1, \ldots, k$; then $T_{\Omega, \alpha}^{A_{1}, \ldots, A_{k}}$ is bounded from $L^{p, \kappa}\left(\omega^{p}, \omega^{q}\right)\left(\mathbb{R}^{n}\right)$ to $L^{q, \kappa q / p}\left(\omega^{q}, \mathbb{R}^{n}\right)$ and

$$
\begin{aligned}
& \left\|T_{\Omega, \alpha}^{A_{1}, \ldots, A_{k}} f\right\|_{L^{q, \kappa q / p}\left(\omega^{q}, \mathbb{R}^{n}\right)} \\
& \quad \leq C\left(\prod_{j=1}^{k} \sum_{|\gamma|=m_{j}-1}\left\|D^{\gamma} A_{j}\right\|_{*}\right)\|f\|_{L^{p, \kappa}\left(\omega^{p}, \omega^{q}\right)\left(\mathbb{R}^{n}\right)} .
\end{aligned}
$$

Remark 9. In Corollary 8 , if $k=1$ and $m_{1}=1$, then we obtain the main result in [12]; if $\Omega=1$ and $\omega=1$, then we obtain the main result in [13].

\section{Some Preliminaries}

We begin with some properties of $A_{p}\left(\mathbb{R}^{n}\right)$ weights which play a great role in the proofs of our main results.

Weight $\omega$ is a nonnegative, locally integrable function on $\mathbb{R}^{n}$. Let $B=B\left(x_{0}, r_{B}\right)$ denote the ball with the center $x_{0}$ and radius $r_{B}$ and let $\lambda B=B\left(x_{0}, \lambda r_{B}\right)$ for any $\lambda>0$. For a given weight function $\omega$ and a measurable set $E$, we also denote the Lebesgue measure of $E$ by $|E|$ and set weighted measure $\omega(E)=\int_{E} \omega(x) d x$. For any given weight function $\omega$ on $\mathbb{R}^{n}$, $X \subseteq \mathbb{R}^{n}$, and $0<p<\infty$, denote by $L^{p}(\omega, X)$ the space of all functions $f$ satisfying

$$
\|f\|_{L^{p}(\omega, X)}=\left(\int_{X}|f(x)|^{p} \omega(x) d x\right)^{1 / p}<\infty .
$$

Weight $\omega$ is said to belong to $A_{p}\left(\mathbb{R}^{n}\right)$ for $1<p<\infty$, if there exists a constant $C$ such that

$$
\left(\frac{1}{|B|} \int_{B} \omega(x) d x\right)\left(\frac{1}{|B|} \int_{B} \omega(x)^{1-p^{\prime}} d x\right)^{p-1} \leq C .
$$

$A_{1}\left(\mathbb{R}^{n}\right)$ denotes the set consisting of all weight functions $\omega$ :

$$
\frac{1}{|B|} \int_{B} \omega(y) d y \leq C \cdot \underset{x \in B}{\operatorname{ess} \inf } \omega(x)
$$

for every ball $B \subset \mathbb{R}^{n}$.

Weight $\omega$ is said to belong to $A_{\infty}\left(\mathbb{R}^{n}\right)$ if there are positive numbers $C$ and $\delta$ such that

$$
\frac{\omega(E)}{\omega(B)} \leq C\left(\frac{|E|}{|B|}\right)^{\delta}
$$

for all balls $B$ and all measurable $E \subset B$. It is well known that

$$
A_{\infty}\left(\mathbb{R}^{n}\right)=\bigcup_{1 \leq p<\infty} A_{p}\left(\mathbb{R}^{n}\right) .
$$

The classical $A_{p}$ weight theory was first introduced by Muckenhoupt in the study of weighted $L^{p}$-boundedness of Hardy-Littlewood maximal function in [14].

Lemma 10 (see $[14,15])$. Suppose $\omega \in A_{p}\left(\mathbb{R}^{n}\right)$. The following statements hold:

(i) For any $1 \leq p<\infty$, there is a positive number $C$ such that

$$
\frac{\omega\left(B_{k}\right)}{\omega\left(B_{j}\right)} \leq C 2^{n p(k-j)} \quad \text { for } k>j .
$$

(ii) For any $1 \leq p<\infty$, there is a positive number $C$ and $\delta$ such that

$$
\frac{\omega\left(B_{k}\right)}{\omega\left(B_{j}\right)} \geq C 2^{\delta(k-j)} \quad \text { for } k>j .
$$

(iii) For any $1<p<\infty$, one has $\omega^{1-p^{\prime}} \in A_{p^{\prime}}$.

We also need another weight class $A(p, q)$ introduced by Muckenhoupt and Wheeden in [16] to study weighted boundedness of fractional integral operators.

Given $1 \leq p \leq q<\infty$, we say that $\omega \in A(p, q)$ if there exists a constant $C$ such that, for every ball $B \subset \mathbb{R}^{n}$, the inequality

$$
\left(\frac{1}{|B|} \int_{B} \omega(y)^{-p^{\prime}} d y\right)^{1 / p^{\prime}}\left(\frac{1}{|B|} \int_{B} \omega(y)^{q} d y\right)^{1 / q} \leq C
$$

holds when $1<p<\infty$, and for every ball $B \subset \mathbb{R}^{n}$ the inequality

$$
\left(\frac{1}{|B|} \int_{B} \omega(y)^{q} d y\right)^{1 / q} \leq C \cdot \underset{x \in B}{\operatorname{ess} \inf } w(x)
$$

holds when $p=1$. 
By (26), we have

$$
\begin{aligned}
& \left(\int_{B} \omega(y)^{-p^{\prime}} d y\right)^{1 / p^{\prime}}\left(\int_{B} \omega(y)^{q} d y\right)^{1 / q} \\
& \quad \leq C|B|^{1 / p^{\prime}+1 / q} .
\end{aligned}
$$

We summarize some properties about weights $A(p, q)$; see $[15,16]$.

Lemma 11. Given $1 \leq p \leq q<\infty$

(i) $\omega \in A(p, q)$ if and only if $\omega^{q} \in A_{1+q / p^{\prime}}$;

(ii) $\omega \in A(p, q)$ if and only if $\omega^{-p^{\prime}} \in A_{1+p^{\prime} / q}$;

(iii) if $p_{1}<p_{2}$ and $q_{2}>q_{1}$, then $A\left(p_{1}, q_{1}\right) \subset A\left(p_{2}, q_{2}\right)$.

John and Nirenberg introduced the function space of $\mathrm{BMO}$ in [17]. A locally integrable function $b$ is said to be in $\operatorname{BMO}\left(\mathbb{R}^{n}\right)$ if

$$
\sup _{B \subset \mathbb{R}^{n}} \frac{1}{|B|} \int_{B}\left|b(x)-b_{B}\right| d x=\|b\|_{*}<\infty,
$$

where

$$
b_{B}=\frac{1}{|B|} \int_{B} b(y) d y
$$

Lemma 12 (see [10]). Suppose $\omega \in A_{\infty}$ and $b \in B M O\left(\mathbb{R}^{n}\right)$. Then for any $1 \leq p<\infty$ and $r_{1}, r_{2}>0$, we have

$$
\begin{aligned}
& \left(\frac{1}{\omega\left(B\left(x_{0}, r_{1}\right)\right)}\right. \\
& \left.\cdot \int_{B\left(x_{0}, r_{1}\right)}\left|b(x)-b_{B\left(x_{0}, r_{2}\right)}\right|^{p} \omega(x) d x\right)^{1 / p} \leq C\|b\|_{*} \\
& \cdot\left(1+\left|\ln \frac{r_{1}}{r_{2}}\right|\right) .
\end{aligned}
$$

Below we recall some conclusions about $R_{m}(A ; x, y)$.

Lemma 13 (see [9]). Let $b$ be a function on $\mathbb{R}^{n}$ with the mth derivatives in $L^{q}\left(\mathbb{R}^{n}\right), q>n$. Then

$$
\begin{aligned}
& \left|R_{m}(b ; x, y)\right| \leq C|x-y|^{m} \sum_{|\gamma|=m}\left(\frac{1}{B(x, 5 \sqrt{n}|x-y|)}\right. \\
& \left.\quad \cdot \int_{B(x, 5 \sqrt{n}|x-y|)}\left|D^{\gamma} b(z)\right|^{q} d z\right)^{1 / q} .
\end{aligned}
$$

Lemma 14 (see [18]). For fixed $x \in \mathbb{R}^{n}$, let

$$
\bar{A}(x)=A(x)-\sum_{|\gamma|=m-1} \frac{1}{\gamma !}\left(D^{\gamma} A\right)_{B(x, 5 \sqrt{n}|x-y|)} x^{\gamma} .
$$

Then $R_{m}(A ; x, y)=R_{m}(\bar{A} ; x, y)$.
Lemma 15. Let $x \in B\left(x_{0}, l\right)$, and let $y \in B\left(x_{0}, 2^{j+1} l\right) \backslash$ $B\left(x_{0}, 2^{j} l\right)$. Then

$$
\begin{aligned}
& \left|R_{m}(A ; x, y)\right| \leq C|x-y|^{m-1}\left(j \sum_{|\gamma|=m-1}\left\|D^{\gamma} A\right\|_{*}\right. \\
& \left.\quad+\sum_{|\gamma|=m-1}\left|D^{\gamma} A(y)-\left(D^{\gamma} A\right)_{B\left(x_{0}, l\right)}\right|\right) .
\end{aligned}
$$

Proof. From Lemma 14, we have

$$
\begin{aligned}
\left|R_{m}(A ; x, y)\right|= & \left|R_{m}(\bar{A} ; x, y)\right| \\
\leq & \left|R_{m-1}(\bar{A} ; x, y)\right| \\
& +\sum_{|\gamma|=m-1} \frac{1}{\gamma !}\left|D^{\gamma} \bar{A}(y)\right||x-y|^{m-1} .
\end{aligned}
$$

By Lemma 13,

$$
\left|R_{m-1}(\bar{A} ; x, y)\right| \leq C|x-y|^{m-1} \sum_{|\gamma|=m-1}\left\|D^{\gamma} A\right\|_{*} .
$$

If $x \in B\left(x_{0}, l\right), y \in B\left(x_{0}, 2^{j+1} l\right) \backslash B\left(x_{0}, 2^{j} l\right)$, we can easily see that $2^{j-1} l \leq|x-y|<2^{j+2} l$. We get

$$
\begin{aligned}
B\left(x_{0}, 2^{j-1} l\right) & \subset B(x, 5 \sqrt{n}|x-y|) \\
& \subset 100 \sqrt{n} B\left(x_{0}, 2^{j} l\right) .
\end{aligned}
$$

Then

$$
\frac{\left|100 \sqrt{n} B\left(x_{0}, 2^{j} l\right)\right|}{|B(x, 5 \sqrt{n}|x-y|)|} \leq \frac{\left|100 \sqrt{n} B\left(x_{0}, 2^{j} l\right)\right|}{\left|B\left(x_{0}, 2^{j-1} l\right)\right|} \leq C .
$$

Thus

$$
\begin{aligned}
& \left|\left(D^{\gamma} A\right)_{B(x, 5 \sqrt{n}|x-y|)}-\left(D^{\gamma} A\right)_{B\left(x_{0}, 2^{j} l\right)}\right| \\
& \quad \leq \frac{1}{|B(x, 5 \sqrt{n}|x-y|)|} \\
& \quad \cdot \int_{B(x, 5 \sqrt{n}|x-y|)}\left|D^{\gamma} A(y)-\left(D^{\gamma} A\right)_{B\left(x_{0}, 2^{j} l\right)}\right| d y \\
& \quad \leq \frac{C}{\left|100 \sqrt{n} B\left(x_{0}, 2^{j} l\right)\right|} \\
& \quad \cdot \int_{100 \sqrt{n} B\left(x_{0}, 2^{j} l\right)}\left|D^{\gamma} A(y)-\left(D^{\gamma} A\right)_{B\left(x_{0}, 2^{j} l\right)}\right| d y \\
& \quad \leq C\left\|D^{\gamma} A\right\|_{*} \cdot
\end{aligned}
$$

Note that

$$
\begin{aligned}
& \left|\left(D^{\gamma} A\right)_{B\left(x_{0}, 2^{j} l\right)}-\left(D^{\gamma} A\right)_{B\left(x_{0}, l\right)}\right| \\
& \quad \leq \sum_{k=1}^{j}\left|\left(D^{\gamma} A\right)_{B\left(x_{0}, 2^{k} l\right)}-\left(D^{\gamma} A\right)_{B\left(x_{0}, 2^{k-1} l\right)}\right| \\
& \quad \leq 2^{n} j\left\|D^{\gamma} A\right\|_{*} .
\end{aligned}
$$


Then

$$
\begin{aligned}
& \left|\left(D^{\gamma} A\right)_{B(x, 5 \sqrt{n}|x-y|)}-\left(D^{\gamma} A\right)_{B\left(x_{0}, l\right)}\right| \\
& \leq\left|\left(D^{\gamma} A\right)_{B(x, 5 \sqrt{n}|x-y|)}-\left(D^{\gamma} A\right)_{B\left(x_{0}, 2^{j} l\right)}\right| \\
& \quad+\left|\left(D^{\gamma} A\right)_{B\left(x_{0}, 2^{j} l\right)}-\left(D^{\gamma} A\right)_{B\left(x_{0}, l\right)}\right| \leq C j\left\|D^{\gamma} A\right\|_{*} .
\end{aligned}
$$

Thus

$$
\begin{aligned}
\left|D^{\gamma} \bar{A}(y)\right|= & \left|D^{\gamma} A(y)-\left(D^{\gamma} A\right)_{B(x, 5 \sqrt{n}|x-y|)}\right| \\
\leq & \left|D^{\gamma} A(y)-\left(D^{\gamma} A\right)_{B\left(x_{0}, l\right)}\right| \\
& +\left|\left(D^{\gamma} A\right)_{B(x, 5 \sqrt{n}|x-y|)}-\left(D^{\gamma} A\right)_{B\left(x_{0}, l\right)}\right| \\
\leq & \left|D^{\gamma} A(y)-\left(D^{\gamma} A\right)_{B\left(x_{0}, l\right)}\right|+C j\left\|D^{\gamma} A\right\|_{*} .
\end{aligned}
$$

Combining with (35), (36), and (42), then (34) is proved.

Finally, we recall a relationship between essential supremum and essential infimum.

Lemma 16 (see [19]). Let $f$ be a real-valued nonnegative function and measurable on $E$. Then

$$
(\underset{x \in E}{\operatorname{essinf}} f(x))^{-1}=\underset{x \in E}{\operatorname{ess} \sup } \frac{1}{f(x)}
$$

\section{A Local Estimate}

To prove Theorem 3, we first investigate the following local estimate.

Theorem 17. Let $0<\alpha<n, 1<p<n / \alpha$, let $1 / q=1 / p-\alpha / n$, and let $\Omega$ be homogeneous of degree zero with $\Omega \in L^{s}\left(\mathbb{S}^{n-1}\right)$, $s>1$. Suppose $\omega^{s^{\prime}} \in A\left(p / s^{\prime}, q / s^{\prime}\right)$ and $A_{j}$ have derivatives of order $m_{j}-1$ in $B M O\left(\mathbb{R}^{n}\right), j=1, \ldots, k$; then, for any $l>0$, there is a constant $C$ independent of $f$ such that

$$
\begin{aligned}
& \left\|T_{\Omega, \alpha}^{A_{1}, \ldots, A_{k}}(f)\right\|_{L^{q}\left(\omega^{q}, B\left(x_{0}, l\right)\right)} \\
& \quad \leq C\left(\prod_{j=1}^{k} \sum_{|\gamma|=m_{j}-1}\left\|D^{\gamma} A_{j}\right\|_{*}\right)\left(\omega^{q}\left(B\left(x_{0}, l\right)\right)\right)^{1 / q} \\
& \quad \cdot \int_{2 l}^{\infty}\|f\|_{L^{p}\left(\omega^{p}, B\left(x_{0}, r\right)\right)}\left(\omega^{q}\left(B\left(x_{0}, r\right)\right)\right)^{-1 / q} \frac{1}{r} d r .
\end{aligned}
$$

Proof. To simplify the proof process of Theorem 17 in the following discussion we consider only the case $k=2$. The method can be used to deal with the case $k>2$ without any essential difficulty.
We write $f$ as $f=f_{1}+f_{2}$, where $f_{1}(y)=f(y) \chi_{B\left(x_{0}, 2 l\right)}(y)$, and $\chi_{B\left(x_{0}, 2 l\right)}$ denotes the characteristic function of $B\left(x_{0}, 2 l\right)$. Then

$$
\begin{aligned}
& \left\|T_{\Omega, \alpha}^{A_{1}, A_{2}}(f)\right\|_{L^{p}\left(\omega^{p}, B\left(x_{0}, l\right)\right)} \\
& \leq\left\|T_{\Omega, \alpha}^{A_{1}, A_{2}}\left(f_{1}\right)\right\|_{L^{p}\left(\omega^{p}, B\left(x_{0}, l\right)\right)} \\
& \quad+\left\|T_{\Omega, \alpha}^{A_{1}, A_{2}}\left(f_{2}\right)\right\|_{L^{p}\left(\omega^{p}, B\left(x_{0}, l\right)\right)} .
\end{aligned}
$$

Since $f_{1} \in L^{p}\left(\omega^{p}, \mathbb{R}^{n}\right)$, by the boundedness of $T_{\Omega, \alpha}^{A_{1}, A_{2}}$ from $L^{p}\left(\omega^{p}, \mathbb{R}^{n}\right)$ to $L^{q}\left(\omega^{q}, \mathbb{R}^{n}\right)$ (Theorem 1 ) we get

$$
\begin{aligned}
& \left\|T_{\Omega, \alpha}^{A_{1}, A_{2}}\left(f_{1}\right)\right\|_{L^{q}\left(\omega^{q}, B\left(x_{0}, l\right)\right)} \leq\left\|T_{\Omega, \alpha}^{A_{1}, A_{2}}\left(f_{1}\right)\right\|_{L^{q}\left(\omega^{q}, \mathbb{R}^{n}\right)} \\
& \quad \leq C\left(\prod_{j=1}^{2} \sum_{|\gamma|=m_{j}-1}\left\|D^{\gamma} A_{j}\right\|_{*}\right)\left\|f_{1}\right\|_{L^{p}\left(\omega^{p}, \mathbb{R}^{n}\right)} \\
& \quad=C\left(\prod_{j=1}^{2} \sum_{|\gamma|=m_{j}-1}\left\|D^{\gamma} A_{j}\right\|_{*}\right)\|f\|_{L^{p}\left(\omega^{p}, B\left(x_{0}, 2 l\right)\right)} .
\end{aligned}
$$

Noting that $q>p>1$ and $s^{\prime} p / p^{\prime}\left(p-s^{\prime}\right) \geq 1$, then by Hölder's inequality

$$
\begin{aligned}
1 & \leq\left(\frac{1}{|B|} \int_{B} \omega(y)^{p} d y\right)^{1 / p}\left(\frac{1}{|B|} \int_{B} \omega(y)^{-p^{\prime}} d y\right)^{1 / p^{\prime}} \\
& \leq\left(\frac{1}{|B|} \int_{B} \omega(y)^{q} d y\right)^{1 / q} \\
& \cdot\left(\frac{1}{|B|} \int_{B} \omega(y)^{-s^{\prime} p /\left(p-s^{\prime}\right)} d y\right)^{\left(p-s^{\prime}\right) / s^{\prime} p} .
\end{aligned}
$$

This means

$$
l^{n / s^{\prime}-\alpha} \leq\left(\omega^{q}\left(B\left(x_{0}, l\right)\right)\right)^{1 / q}\left\|\omega^{-1}\right\|_{L^{s^{\prime} p /\left(p-s^{\prime}\right)}\left(B\left(x_{0}, l\right)\right)} .
$$

Thus

$$
\begin{aligned}
& \|f\|_{L^{p}\left(\omega^{p}, B\left(x_{0}, 2 l\right)\right)} \leq C l^{n / s^{\prime}-\alpha}\|f\|_{L^{p}\left(\omega, B\left(x_{0}, 2 l\right)\right)} \\
& \cdot \int_{2 l}^{\infty} \frac{1}{r^{n / s^{\prime}-\alpha+1}} d r \leq C\left(\omega^{q} B\left(x_{0}, l\right)\right)^{1 / q} \\
& \cdot\left\|\omega^{-1}\right\|_{L^{s^{\prime} p /\left(p-s^{\prime}\right)}\left(B\left(x_{0}, l\right)\right)} \int_{2 l}^{\infty}\|f\|_{L^{p}\left(\omega, B\left(x_{0}, r\right)\right)} \\
& \cdot \frac{1}{r^{n / s^{\prime}-\alpha+1}} d r \leq C\left(\omega^{q} B\left(x_{0}, l\right)\right)^{1 / q} \\
& \cdot \int_{2 l}^{\infty}\|f\|_{L^{p}\left(\omega, B\left(x_{0}, r\right)\right)}\left\|\omega^{-1}\right\|_{L^{s^{\prime} p /\left(p-s^{\prime}\right)}\left(B\left(x_{0}, r\right)\right)} \frac{1}{r^{n / s^{\prime}-\alpha+1}} d r .
\end{aligned}
$$

Since $\omega^{s^{\prime}} \in A\left(p / s^{\prime}, q / s^{\prime}\right)$, by (28), we get

$$
\left(\omega^{q}\left(B\left(x_{0}, r\right)\right)\right)^{1 / q}\left\|\omega^{-1}\right\|_{L^{s^{\prime} p /\left(p-s^{\prime}\right)}\left(B\left(x_{0}, r\right)\right)} \leq C r^{n / s^{\prime}-\alpha}
$$


holds for all $r>0$. Then

$$
\begin{aligned}
& \left\|T_{\Omega, \alpha}^{A_{1}, A_{2}}\left(f_{1}\right)\right\|_{L^{q}\left(\omega^{q}, B\left(x_{0}, l\right)\right)} \leq C\left(\prod_{j=1}^{2} \sum_{|\gamma|=m_{j}-1}\left\|D^{\gamma} A_{j}\right\|_{*}\right) \\
& \cdot\left(\omega^{q}\left(B\left(x_{0}, l\right)\right)\right)^{1 / q}
\end{aligned}
$$

$$
\cdot \int_{2 l}^{\infty}\|f\|_{L^{p}\left(\omega^{p}, B\left(x_{0}, r\right)\right)}\left(\omega^{q}\left(B\left(x_{0}, r\right)\right)\right)^{-1 / q} \frac{1}{r} d r .
$$

Let $N=m_{1}+m_{2}-2$, and let $\Delta_{i}=\left(B\left(x_{0}, 2^{j+1} l\right)\right) \backslash$ $\left(B\left(x_{0}, 2^{j} l\right)\right)$. Suppose $x \in B\left(x_{0}, l\right)$. By Lemma 15 ,

$$
\begin{aligned}
\left|T_{\Omega, \alpha}^{A_{1}, A_{2}}\left(f_{2}\right)(x)\right| \leq & \left|\int_{\left(B\left(x_{0}, 2 l\right)\right)^{c}} \frac{\Omega(x-y)}{|x-y|^{n-\alpha+N}} R_{m_{1}}\left(A_{1} ; x, y\right) R_{m_{2}}\left(A_{2} ; x, y\right) f(y) d y\right| \\
\leq & C \sum_{j=1}^{\infty} \int_{\Delta_{i}} \frac{|\Omega(x-y) f(y)|}{|x-y|^{n-\alpha}} \prod_{k=1}^{2}\left(j \sum_{|\gamma|=m_{k}-1}\left\|D^{\gamma} A_{k}\right\|_{*}+\sum_{|\gamma|=m_{k}-1}\left|D^{\gamma} A_{k}(y)-\left(D^{\gamma} A_{k}\right)_{B\left(x_{0}, l\right)}\right|\right) d y \\
\leq & C\left(\prod_{j=1}^{2} \sum_{|\gamma|=m_{j}-1}\left\|D^{\gamma} A_{j}\right\|_{*}\right) \sum_{j=1}^{\infty} j^{2} \int_{\Delta_{i}} \frac{|\Omega(x-y) f(y)|}{|x-y|^{n-\alpha}} d y \\
& +C \sum_{|\gamma|=m_{1}-1}\left\|D^{\gamma} A_{1}\right\|_{*} \sum_{|\gamma|=m_{2}-1} \sum_{j=1}^{\infty} j \int_{\Delta_{i}} \frac{|\Omega(x-y) f(y)|}{|x-y|^{n-\alpha}}\left|D^{\gamma} A_{2}(y)-\left(D^{\gamma} A_{2}\right)_{B\left(x_{0}, l\right)}\right| d y \\
& +C \sum_{|\gamma|=m_{2}-1}\left\|D^{\gamma} A_{2}\right\|_{*} \sum_{|\gamma|=m_{1}-1} \sum_{j=1}^{\infty} j \int_{\Delta_{i}} \frac{|\Omega(x-y) f(y)|}{|x-y|^{n-\alpha}}\left|D^{\gamma} A_{1}(y)-\left(D^{\gamma_{1}} A_{1}\right)_{B\left(x_{0}, l\right)}\right| d y \\
& +C \sum_{|\gamma|=m_{1}-1} \sum_{|\gamma|=m_{2}-1} \sum_{j=1}^{\infty} \int_{\Delta_{i}} \frac{|\Omega(x-y) f(y)|}{|x-y|^{2}}\left|\prod_{k=1}^{n-\alpha} D_{k}(y)-\left(D^{\gamma} A_{k}\right)_{B\left(x_{0}, l\right)}\right| d y=I_{1}+I_{2}+I_{3}+I_{4} .
\end{aligned}
$$

By Hölder's inequalities,

$$
\begin{aligned}
& \int_{\Delta_{i}} \frac{|\Omega(x-y) f(y)|}{|x-y|^{n-\alpha}} d y \leq\left(\int_{\Delta_{i}}|\Omega(x-y)|^{s} d y\right)^{1 / s} \\
& \cdot\left(\int_{\Delta_{i}} \frac{|f(y)|^{s^{\prime}}}{|x-y|^{(n-\alpha) s^{\prime}}} d y\right)^{1 / s^{\prime}} .
\end{aligned}
$$

When $x \in B\left(x_{0}, s\right)$ and $y \in \Delta_{i}$, then by a direct calculation, we can see that $2^{j-1} l \leq|y-x|<2^{j+1} l$. Then

$$
\begin{aligned}
& \left(\int_{\Delta_{i}}|\Omega(x-y)|^{s} d y\right)^{1 / s} \\
& \quad \leq C\|\Omega\|_{L^{s}\left(S^{n-1}\right)}\left|B\left(x_{0}, 2^{j+1} l\right)\right|^{1 / s} .
\end{aligned}
$$

We also note that if $x \in B\left(x_{0}, l\right), y \in B\left(x_{0}, 2 l\right)^{c}$, then $|y-x| \sim$ $\left|y-x_{0}\right|$. Consequently

$$
\begin{aligned}
& \left(\int_{\Delta_{i}} \frac{|f(y)|^{s^{\prime}}}{|x-y|^{(n-\alpha) s^{\prime}}} d y\right)^{1 / s^{\prime}} \\
& \quad \leq \frac{1}{\left|B\left(x_{0}, 2^{j+1} l\right)\right|^{1-\alpha / n}}\left(\int_{B\left(x_{0}, j^{j+1} l\right)}|f(y)|^{s^{\prime}} d y\right)^{1 / s^{\prime}} .
\end{aligned}
$$

Then

$$
\begin{aligned}
I_{1} & \leq C\left(\prod_{j=1}^{2} \sum_{|\gamma|=m_{j}-1}\left\|D^{\gamma} A_{j}\right\|_{*}\right) \\
& \cdot \sum_{j=1}^{\infty} j^{2}\left(2^{j+1} l\right)^{\alpha-n / s^{\prime}}\left(\int_{B\left(x_{0}, 2^{j+1} l\right)}|f(y)|^{s^{\prime}} d y\right)^{1 / s^{\prime}} .
\end{aligned}
$$

Since $s^{\prime}<p$, it follows from Hölder's inequality that

$$
\begin{aligned}
& \left(\int_{B\left(x_{0}, 2^{j+1} l\right)}|f(y)|^{s^{\prime}} d y\right)^{1 / s^{\prime}} \\
& \quad \leq C\|f\|_{\left.L^{p}\left(\omega^{p}, B\left(x_{0}, 2^{j+1} l\right)\right)\right)}\left\|\omega^{-1}\right\|_{L^{s^{\prime} /\left(p-s^{\prime}\right)}\left(B\left(x_{0}, 2^{j+1} l\right)\right)} .
\end{aligned}
$$

Then

$$
\begin{gathered}
\sum_{j=1}^{\infty} j^{2}\left(2^{j+1} l\right)^{\alpha-n / s^{\prime}}\left(\int_{B\left(x_{0}, j^{j+1} s\right)}|f(y)|^{s^{\prime}} d y\right)^{1 / s^{\prime}} \\
\leq C \sum_{j=1}^{\infty}\left(1+\ln \frac{2^{j+1} l}{l}\right)^{2}\left(2^{j+1} l\right)^{\alpha-n / s^{\prime}} \\
\cdot\|f\|_{L^{p}\left(\omega^{p}, B\left(x_{0}, 2^{j+1} l\right)\right)}\left\|\omega^{-1}\right\|_{L^{s^{\prime} p /\left(p-s^{\prime}\right)}\left(B\left(x_{0}, 2^{j+1} l\right)\right)}
\end{gathered}
$$




$$
\begin{aligned}
& \leq C \sum_{j=1}^{\infty} \int_{2^{j+1} l}^{2^{j+2} l}\left(1+\ln \frac{r}{l}\right)^{2}\|f\|_{L^{p}\left(\omega^{p}, B\left(x_{0}, r\right)\right)} \\
& \cdot\left\|\omega^{-1}\right\|_{L^{s^{\prime} p /\left(p-s^{\prime}\right)}\left(B\left(x_{0}, r\right)\right)} \frac{1}{r^{1-\alpha+n / s^{\prime}}} d r \leq C \int_{2 l}^{\infty}(1 \\
& \left.+\ln \frac{r}{l}\right)^{2}\|f\|_{L^{p}\left(\omega^{p}, B\left(x_{0}, r\right)\right)}\left\|\omega^{-1}\right\|_{L^{s^{\prime} p /\left(p-s^{\prime}\right)}\left(B\left(x_{0}, r\right)\right)} \\
& \cdot \frac{1}{r^{1-\alpha+n / s^{\prime}}} d r .
\end{aligned}
$$

From (50) we know

$$
\left\|\omega^{-1}\right\|_{L^{s^{\prime} p /\left(p-s^{\prime}\right)}\left(B\left(x_{0}, r\right)\right)} \leq C r^{n / s^{\prime}-\alpha}\left(\omega^{q}\left(B\left(x_{0}, r\right)\right)\right)^{-1 / q} .
$$

Then

$$
\begin{aligned}
& I_{1} \leq C\left(\prod_{j=1}^{2} \sum_{|\gamma|=m_{j}-1}\left\|D^{\gamma} A_{j}\right\|_{*}\right) \int_{2 l}^{\infty}\left(1+\ln \frac{r}{l}\right)^{2} \\
& \cdot\|f\|_{L^{p}\left(\omega^{p}, B\left(x_{0}, r\right)\right)}\left(\omega^{q}\left(B\left(x_{0}, r\right)\right)\right)^{-1 / q} \frac{1}{r} d r .
\end{aligned}
$$

By Hölder's inequality and (54), (55) we have

$$
\begin{aligned}
& \int_{\Delta_{i}} \frac{|\Omega(x-y) f(y)|}{|x-y|^{n-\alpha}}\left|D^{\gamma} A_{2}(y)-\left(D^{\gamma} A_{2}\right)_{B\left(x_{0}, l\right)}\right| d y \\
& \leq\left(\int_{\Delta_{i}}|\Omega(x-y)|^{s} d y\right)^{1 / s} \\
& \cdot\left(\int_{\Delta_{i}} \frac{\left|\left(D^{\gamma} A_{2}(y)-\left(D^{\gamma} A_{2}\right)_{B\left(x_{0}, l\right)}\right) f(y)\right|^{s^{\prime}}}{|x-y|^{(n-\alpha) s^{\prime}}} d y\right)^{1 / s^{\prime}} \\
& \quad \leq C \sum_{j=1}^{\infty}\left(2^{j+1} l\right)^{\alpha-n / s^{\prime}} \\
& \cdot\left(\int_{B\left(x_{0}, 2^{j+1} l\right)}\left|D^{\gamma} A_{2}(y)-\left(D^{\gamma} A_{2}\right)_{B\left(x_{0}, l\right)}\right|^{s^{\prime}}\right. \\
& \left.\cdot|f(y)|^{s^{\prime}} d y\right)^{1 / s^{\prime}} \cdot
\end{aligned}
$$

Applying Hölder's inequality again, we get

$$
\begin{aligned}
& \left(\int_{B\left(x_{0}, 2^{j+1} l\right)}\left|D^{\gamma} A_{2}(y)-\left(D^{\gamma} A_{2}\right)_{B\left(x_{0}, l\right)}\right|^{s^{\prime}}\right. \\
& \left.\cdot|f(y)|^{s^{\prime}} d y\right)^{1 / s^{\prime}} \leq C\|f\|_{L^{p}\left(\omega^{p}, B\left(x_{0}, 2^{j+1} l\right)\right)} \\
& \cdot \|\left(D^{\gamma} A_{2}(y)-\left(D^{\gamma} A_{2}\right)_{B\left(x_{0}, l\right)}\right) \\
& \cdot \omega(\cdot)^{-1} \|_{L^{p s^{\prime} /\left(p-s^{\prime}\right)}\left(B\left(x_{0}, 2^{j+1} l\right)\right)} \cdot
\end{aligned}
$$

Consequently,

$$
\begin{aligned}
I_{2} & \leq C \sum_{|\gamma|=m_{1}-1}\left\|D^{\gamma} A_{1}\right\|_{*} \sum_{|\gamma|=m_{2}-1} \sum_{j=1}^{\infty} \int_{2^{j+1} l}^{2^{j+2} l}\left(1+\ln \frac{2^{j+1} l}{l}\right) \\
& \cdot\left(2^{j+1} l\right)^{\alpha-n / s^{\prime}}\|f\|_{L^{p}\left(\omega^{p}, B\left(x_{0}, r\right)\right)} \\
& \cdot\left\|\left(D^{\gamma} A_{2}(y)-\left(D^{\gamma} A_{2}\right)_{B\left(x_{0}, l\right)}\right) \omega(\cdot)^{-1}\right\|_{L^{p s^{\prime} /\left(p-s^{\prime}\right)}\left(B\left(x_{0}, r\right)\right)} d r \\
& \leq C \sum_{|\gamma|=m_{1}-1}\left\|D^{\gamma} A_{1}\right\|_{*} \sum_{|\gamma|=m_{2}-1} \int_{2 l}^{\infty}\|f\|_{L^{p}\left(\omega^{p}, B\left(x_{0}, r\right)\right)} \\
& \cdot\left\|\left(D^{\gamma} A_{2}(y)-\left(D^{\gamma} A_{2}\right)_{B\left(x_{0}, l\right)}\right) \omega(\cdot)^{-1}\right\|_{L^{p s^{\prime} /\left(p-s^{\prime}\right)}\left(B\left(x_{0}, r\right)\right)} \\
& \cdot \frac{1}{r^{1-\alpha+n / s^{\prime}}} d r .
\end{aligned}
$$

By $\omega^{s^{\prime}} \in A\left(p / s^{\prime}, q / s^{\prime}\right)$ and (ii) of Lemma 11 we know $\omega^{-s^{\prime} p /\left(p-s^{\prime}\right)} \in A_{1+p s^{\prime} /\left(p-s^{\prime}\right) q}$. Then it follows from (31) and (59) that

$$
\begin{aligned}
& \left\|\left(D^{\gamma} A_{2}(y)-\left(D^{\gamma} A_{2}\right)_{B\left(x_{0}, l\right)}\right) \omega(\cdot)^{-1}\right\|_{L^{p s^{\prime} /\left(p-s^{\prime}\right)}\left(B\left(x_{0}, r\right)\right)} \\
& \leq\left(\int_{B\left(x_{0}, r\right)}\left|\left(D^{\gamma} A_{2}(y)-\left(D^{\gamma} A_{2}\right)_{B\left(x_{0}, l\right)}\right)\right|^{p s^{\prime} /\left(p-s^{\prime}\right)}\right. \\
& \left.\cdot \omega^{-p s^{\prime} /\left(p-s^{\prime}\right)}(y) d y\right)^{\left(p-s^{\prime}\right) / p s^{\prime}} \leq C\left\|D^{\gamma} A_{2}\right\|_{*}\left(1+\ln \frac{r}{l}\right) \\
& \cdot\left(\omega^{-p s^{\prime} /\left(p-s^{\prime}\right)}\left(B\left(x_{0}, r\right)\right)\right)^{\left(p-s^{\prime}\right) / p s^{\prime}}=C\left\|D^{\gamma} A_{2}\right\|_{*}(1 \\
& \left.+\ln \frac{r}{l}\right)\left\|\omega^{-1}\right\|_{L^{p s^{\prime} /\left(p-s^{\prime}\right)}\left(B\left(x_{0}, r\right)\right)} \leq C\left\|D^{\gamma} A_{2}\right\|_{*}\left(1+\ln \frac{r}{l}\right) \\
& \cdot r^{n / s^{\prime}-\alpha}\left(\omega^{q}\left(B\left(x_{0}, r\right)\right)\right)^{-1 / q} .
\end{aligned}
$$

Hence

$$
\begin{gathered}
I_{2} \leq C\left(\prod_{j=1}^{2} \sum_{|\gamma|=m_{j}-1}\left\|D^{\gamma} A_{j}\right\|_{*}\right) \int_{2 l}^{\infty}\left(1+\ln \frac{r}{l}\right) \\
\cdot\|f\|_{L^{p}\left(\omega^{p}, B\left(x_{0}, r\right)\right)}\left(\omega^{q}\left(B\left(x_{0}, r\right)\right)\right)^{-1 / q} \frac{1}{r} d r .
\end{gathered}
$$

Similar to the estimates for $I_{2}$, we have

$$
\begin{gathered}
I_{3} \leq C\left(\prod_{j=1}^{2} \sum_{|\gamma|=m_{j}-1}\left\|D^{\gamma} A_{j}\right\|_{*}\right) \int_{2 l}^{\infty}\left(1+\ln \frac{r}{l}\right) \\
\cdot\|f\|_{L^{p}\left(\omega^{p}, B\left(x_{0}, r\right)\right)}\left(\omega^{q}\left(B\left(x_{0}, r\right)\right)\right)^{-1 / q} \frac{1}{r} d r .
\end{gathered}
$$

Finally, we come to estimate $I_{4}$. 
Similar to (59), we get

$$
\begin{aligned}
& \int_{\Delta_{i}} \frac{|\Omega(x-y) f(y)|}{|x-y|^{n-\alpha}} \prod_{k=1}^{2}\left|D^{\gamma} A_{k}(y)-\left(D^{\gamma} A_{k}\right)_{B\left(x_{0}, l\right)}\right| d y \\
& \leq C\left(2^{j+1} l\right)^{\alpha-n / s^{\prime}} \\
& \cdot\left(\int_{B\left(x_{0}, 2^{j+1} l\right)}\left|\prod_{k=1}^{2}\left(D^{\gamma} A_{k}(y)-\left(D^{\gamma} A_{k}\right)_{B\left(x_{0}, l\right)}\right)\right|^{s^{\prime}}\right. \\
& \left.\cdot|f(y)|^{s^{\prime}} d y\right)^{1 / s^{\prime}} .
\end{aligned}
$$

By Hölder's inequality we get

$$
\begin{aligned}
& \left(\int_{B\left(x_{0}, 2^{j+1} l\right)}\left|\prod_{k=1}^{2}\left(D^{\gamma} A_{k}(y)-\left(D^{\gamma} A_{k}\right)_{B\left(x_{0}, l\right)}\right)\right|^{s^{\prime}}\right. \\
& \left.\cdot|f(y)|^{s^{\prime}} d y\right)^{1 / s^{\prime}} \leq C\|f\|_{L^{p}\left(\omega^{p}, B\left(x_{0}, 2^{j+1} l\right)\right)} \\
& \cdot \|\left(\prod_{k=1}^{2}\left(D^{\gamma} A_{k}(y)-\left(D^{\gamma} A_{k}\right)_{B\left(x_{0}, l\right)}\right)\right) \\
& \cdot \omega(\cdot)^{-1}\left\|_{L^{p s^{\prime} /\left(p-s^{\prime}\right)}\left(B\left(x_{0}, 2^{j+1} l\right)\right)} \leq C\right\| f \|_{L^{p}\left(\omega^{p}, B\left(x_{0}, 2^{j+1} l\right)\right)} \\
& \cdot \prod_{k=1}^{2} \| D^{\gamma} A_{k}(y)-\left(D^{\gamma} A_{k}\right)_{B\left(x_{0}, l\right)} \mid \\
& \cdot \omega(\cdot)^{-1 / 2} \|_{L^{2 p s^{\prime} /\left(p-s^{\prime}\right)}\left(B\left(x_{0}, 2^{j+1} l\right)\right)} \cdot
\end{aligned}
$$

Then

$$
\begin{aligned}
& \sum_{j=1}^{\infty} \int_{\Delta_{i}} \frac{|\Omega(x-y) f(y)|}{|x-y|^{n-\alpha}} \prod_{k=1}^{2}\left|D^{\gamma} A_{k}(y)-\left(D^{\gamma} A_{k}\right)_{B\left(x_{0}, l\right)}\right| d y \\
& \leq C \int_{2 l}^{\infty}\|f\|_{L^{p}\left(\omega^{p}, B\left(x_{0}, r\right)\right)} \prod_{k=1}^{2} \|\left|D^{\gamma} A_{k}(y)-\left(D^{\gamma} A_{k}\right)_{B\left(x_{0}, l\right)}\right| \\
& \cdot \omega(\cdot)^{-1 / 2} \|_{L^{2 p s^{\prime} /\left(p-s^{\prime}\right)}\left(B\left(x_{0}, r\right)\right)} \frac{1}{r^{1-\alpha+n / s^{\prime}}} d r .
\end{aligned}
$$

Since $\omega^{-s^{\prime} p /\left(p-s^{\prime}\right)} \in A_{1+p s^{\prime} /\left(p-s^{\prime}\right) q}$, similar to (64), we can deduce

$$
\begin{aligned}
& \|\left|D^{\gamma} A_{k}(y)-\left(D^{\gamma} A_{k}\right)_{B\left(x_{0}, l\right)}\right| \\
& \cdot \omega(\cdot)^{-1 / 2}\left\|_{L^{2 p s^{\prime} /\left(p-s^{\prime}\right)}\left(B\left(x_{0}, 2^{j+1} l\right)\right)} \leq C\right\| D^{\gamma} A_{k} \|_{*}(1 \\
& \left.+\ln \frac{r}{l}\right)\left(\omega^{-p s^{\prime} /\left(p-s^{\prime}\right)}\left(B\left(x_{0}, r\right)\right)\right)^{\left(p-s^{\prime}\right) / 2 p s^{\prime}} .
\end{aligned}
$$

Then by (70) we get

$$
\begin{aligned}
& \sum_{j=1}^{\infty} \int_{\Delta_{i}} \frac{|\Omega(x-y) f(y)|}{|x-y|^{n-\alpha}} \prod_{k=1}^{2}\left|D^{\gamma} A_{k}(y)-\left(D^{\gamma} A_{k}\right)_{B\left(x_{0}, l\right)}\right| d y \\
& \quad \leq C \prod_{k=1}^{2}\left\|D^{\gamma} A_{k}\right\|_{*} \int_{2 l}^{\infty}\left(1+\ln \frac{r}{l}\right)^{2}\|f\|_{L^{p}\left(\omega^{p}, B\left(x_{0}, r\right)\right)} \\
& \cdot\left(\omega^{q}\left(B\left(x_{0}, r\right)\right)\right)^{-1 / q} \frac{1}{r} d r .
\end{aligned}
$$

Thus

$$
\begin{gathered}
I_{4} \leq C\left(\prod_{j=1}^{2} \sum_{|\gamma|=m_{j}-1}\left\|D^{\gamma} A_{j}\right\|_{*}\right) \int_{2 l}^{\infty}\left(1+\ln \frac{r}{l}\right) \\
\cdot\|f\|_{L^{p}\left(\omega^{p}, B\left(x_{0}, r\right)\right)}\left(\omega^{q}\left(B\left(x_{0}, r\right)\right)\right)^{-1 / q} \frac{1}{r} d r .
\end{gathered}
$$

Combining the estimates for $I_{1}, I_{2}, I_{3}$, and $I_{4}$, we get

$$
\begin{aligned}
& \left|T_{\Omega, \alpha}^{A_{1}, A_{2}}\left(f_{2}\right)(x)\right| \leq C\left(\prod_{j=1}^{2} \sum_{|\gamma|=m_{j}-1}\left\|D^{\gamma} A_{j}\right\|_{*}\right) \\
& \cdot \int_{2 l}^{\infty}\left(1+\ln \frac{r}{l}\right)\|f\|_{L^{p}\left(\omega^{p}, B\left(x_{0}, r\right)\right)}\left(\omega^{q}\left(B\left(x_{0}, r\right)\right)\right)^{-1 / q} \\
& \quad \cdot \frac{1}{r} d r .
\end{aligned}
$$

Then

$$
\begin{gathered}
\left\|T_{\Omega, \alpha}^{A_{1}, A_{2}}\left(f_{2}\right)\right\|_{L^{p}\left(\omega^{p}, B\left(x_{0}, l\right)\right)} \leq C\left(\prod_{j=1}^{2} \sum_{|\gamma|=m_{j}-1}\left\|D^{\gamma} A_{j}\right\|_{*}\right) \\
\cdot\left(\omega^{q}\left(B\left(x_{0}, l\right)\right)\right)^{1 / q} \\
\cdot \int_{2 l}^{\infty}\|f\|_{L^{p}\left(\omega^{p}, B\left(x_{0}, r\right)\right)}\left(\omega^{q}\left(B\left(x_{0}, r\right)\right)\right)^{-1 / q} \frac{1}{r} d r .
\end{gathered}
$$

This completes the proof of Theorem 17.

\section{Proof of Theorem 3}

Since $f \in M_{\varphi_{1}}^{p}\left(\omega^{p}, \mathbb{R}^{n}\right)$, then by Lemma 16 and the fact that $\|f\|_{L^{p}\left(\omega^{p}, B\left(x_{0}, r\right)\right)}$ is a nondecreasing function of $r$, we get

$$
\begin{aligned}
& \frac{\|f\|_{L^{p}\left(\omega^{p}, B\left(x_{0}, r\right)\right)}}{\text { ess } \inf _{0<r<t<\infty} \varphi_{1}\left(x_{0}, t\right)\left(\omega^{p}\left(B\left(x_{0}, t\right)\right)\right)^{1 / p}} \\
& \leq \operatorname{ess} \sup _{0<r<t<\infty} \frac{\|f\|_{L^{p}\left(\omega^{p}, B\left(x_{0}, r\right)\right)}}{\varphi_{1}\left(x_{0}, t\right)\left(\omega^{p}\left(B\left(x_{0}, t\right)\right)\right)^{1 / p}} \\
& \leq \sup _{t>0, x_{0} \in \mathbb{R}^{n}} \frac{\|f\|_{L^{p}\left(\omega^{p}, B\left(x_{0}, t\right)\right)}\left(x_{0}, t\right)\left(\omega^{p}\left(B\left(x_{0}, t\right)\right)\right)^{1 / p}}{\leq\|f\|_{M_{\varphi_{1}}^{p}\left(\omega^{p}, \mathbb{R}^{n}\right)} \cdot}
\end{aligned}
$$


Since $\left(\varphi_{1}, \varphi_{2}\right)$ satisfies (15), we have

$$
\begin{aligned}
& \int_{l}^{\infty}\left(1+\ln \frac{r}{l}\right)^{k}\|f\|_{L^{p}\left(\omega^{p}, B\left(x_{0}, r\right)\right)}\left(\omega^{q}\left(B\left(x_{0}, r\right)\right)\right)^{-1 / q} \\
& \cdot \frac{1}{r} d r \\
& \leq \int_{l}^{\infty} \frac{\|f\|_{L^{p}\left(\omega^{p}, B\left(x_{0}, r\right)\right)}}{\operatorname{ess} \inf _{r<t<\infty} \varphi_{1}\left(x_{0}, t\right)\left(\omega^{p}\left(B\left(x_{0}, t\right)\right)\right)^{1 / p}}(1 \\
& \left.+\ln \frac{r}{l}\right)^{k} \\
& . \frac{\operatorname{ess} \inf _{r<t<\infty} \varphi_{1}\left(x_{0}, t\right)\left(\omega^{p}\left(B\left(x_{0}, t\right)\right)\right)^{1 / p}}{\left(\omega^{q}\left(B\left(x_{0}, r\right)\right)\right)^{1 / q}} \frac{1}{r} d r \\
& \leq C\|f\|_{M_{\varphi_{1}}^{p}\left(\omega^{p}, \mathbb{R}^{n}\right)} \int_{l}^{\infty}\left(1+\ln \frac{r}{l}\right)^{k} \\
& . \\
& \leq C\|f\|_{M_{\varphi_{1}}^{p}\left(\omega^{p}, \mathbb{R}^{n}\right)} \varphi_{2}\left(x_{0}, l\right) . \\
& \quad \frac{\operatorname{ess} \inf _{r<t<\infty} \varphi_{1}\left(x_{0}, t\right)\left(\omega^{p}\left(B\left(x_{0}, t\right)\right)\right)^{1 / p}}{1} \frac{1}{r} d r
\end{aligned}
$$

Then by Theorem 17 we get

$$
\begin{aligned}
& \left\|T_{\Omega, \alpha}^{A_{1}, \ldots, A_{k}}(f)\right\|_{M_{\varphi_{2}}^{q}\left(\omega^{q}, \mathbb{R}^{n}\right)} \leq C \sup _{x_{0} \in \mathbb{R}^{n}, l>0} \frac{1}{\varphi_{2}\left(x_{0}, l\right)} \\
& \cdot\left(\frac{1}{\omega^{q} B\left(x_{0}, l\right)}\right. \\
& \left.\cdot \int_{B\left(x_{0}, l\right)}\left|T_{\Omega, \alpha}^{A_{1}, \ldots, A_{k}}(f)(y)\right|^{q} \omega^{q}(y) d y\right)^{1 / q} \\
& \leq C\left(\prod_{j=1}^{k} \sum_{|\gamma|=m_{j}-1}\left\|D^{\gamma} A\right\|_{*}\right)_{x_{0} \in \mathbb{R}^{n}, l>0} \frac{1}{\varphi_{2}\left(x_{0}, l\right)} \\
& \cdot \int_{l}^{\infty}\left(1+\ln \frac{r}{l}\right)^{k}\|f\|_{L^{p}\left(\omega^{p}, B\left(x_{0}, r\right)\right)}\left(\omega^{q}\left(B\left(x_{0}, r\right)\right)\right)^{-1 / q} \\
& \quad \frac{1}{r} d r \leq C\left(\prod_{j=1}^{k} \sum_{|\gamma|=m_{j}-1}\left\|D^{\gamma} A\right\|_{*}\right)\|f\|_{M_{\varphi_{1}}^{p}\left(\omega^{p}, \mathbb{R}^{n}\right)} .
\end{aligned}
$$

\section{Conflict of Interests}

The authors declare that they have no conflict of interests.

\section{Acknowledgments}

The authors are very grateful to the anonymous referees and the editor for their insightful comments and suggestions.

\section{References}

[1] Q. Wu and D. Yang, "On fractional multilinear singular integrals," Mathematische Nachrichten, vol. 239/240, pp. 215-235, 2002.
[2] Y. Ding and S. Lu, "Higher order commutators for a class of rough operators," Arkiv för Matematik, vol. 37, no. 1, pp. 33-44, 1999.

[3] Y. Ding and S. Z. Lu, "Weighted boundedness for a class of rough multilinear operators," Acta Mathematica Sinica, vol. 17, no. 3, pp. 517-526, 2001.

[4] C. B. Morrey, "On the solutions of quasi-linear elliptic partial differential equations," Transactions of the American Mathematical Society, vol. 43, no. 1, pp. 126-166, 1938.

[5] D. K. Palagachev and L. G. Softova, "Singular integral operators, Morrey spaces and fine regularity of solutions to PDE's," Potential Analysis, vol. 20, no. 3, pp. 237-263, 2004.

[6] G. Di Fazio and M. A. Ragusa, "Interior estimates in Morrey spaces for strong solutions to nondivergence form equations with discontinuous coefficients," Journal of Functional Analysis, vol. 112, no. 2, pp. 241-256, 1993.

[7] M. A. Ragusa, "Regularity of solutions of divergence form elliptic equations," Proceedings of the American Mathematical Society, vol. 128, no. 2, pp. 533-540, 2000.

[8] T. Mizuhara, "Boundedness of some classical operators on generalized Morrey spaces," in ICM-90 Satellite Conference Proceedings: Harmonic Analysis, S. Igari, Ed., pp. 183-189, Springer Japan, 1991.

[9] Y. Komori and S. Shirai, "Weighted Morrey spaces and a singular integral operator," Mathematische Nachrichten, vol. 282, no. 2, pp. 219-231, 2009.

[10] Y. Hu and Y. Wang, "Multilinear fractional integral operators on generalized weighted Morrey spaces," Journal of Inequalities and Applications, vol. 2014, article 323, 18 pages, 2014.

[11] S. Lu, D. Yang, and Z. Zhou, "Sublinear operators with rough kernel on generalized morrey spaces," Hokkaido Mathematical Journal, vol. 27, no. 1, pp. 219-232, 1998.

[12] H. Wang, "Boundedness of fractional integral operators with rough kernels on weighted Morrey spaces," Acta Mathematica Sinica, vol. 56, no. 2, pp. 175-186, 2013.

[13] L. Liu, "Boundedness for multilinear singular integral operators on Morrey spaces," Bulletin of the Malaysian Mathematical Sciences Society, vol. 33, no. 1, pp. 93-103, 2010.

[14] B. Muckenhoupt, "Weighted norm inequalities for the Hardy maximal function," Transactions of the American Mathematical Society, vol. 165, pp. 207-226, 1972.

[15] J. Garcia-Cuerva and J. L. Rubio de Francia, Weighted Norm Inequalities and Related Topics, North-Holland, Amsterdam, The Netherlands, 1985.

[16] B. Muckenhoupt and R. L. Wheeden, "Weighted norm inequalities for singular and fractional integrals," Transactions of the American Mathematical Society, vol. 161, pp. 249-258, 1971.

[17] F. John and L. Nirenberg, "On functions of bounded mean oscillation," Communications on Pure and Applied Mathematics, vol. 14, pp. 415-426, 1961.

[18] J. Cohen and J. Gosselin, "A BMO estimate for multilinear singular integrals," Illinois Journal of Mathematics, vol. 30, no. 3, pp. 445-464, 1986.

[19] R. Wheeden and A. Zygmund, Measure and Integral: An Introduction to Real Analysis, vol. 43 of Pure and Applied Mathematics, Marcel Dekker, New York, NY, USA, 1977. 


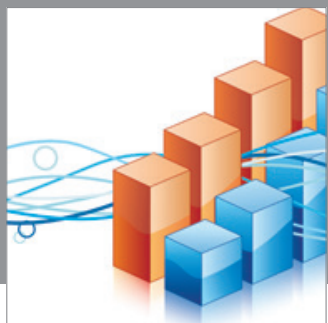

Advances in

Operations Research

mansans

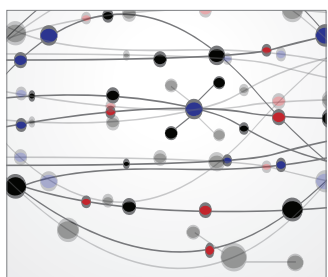

The Scientific World Journal
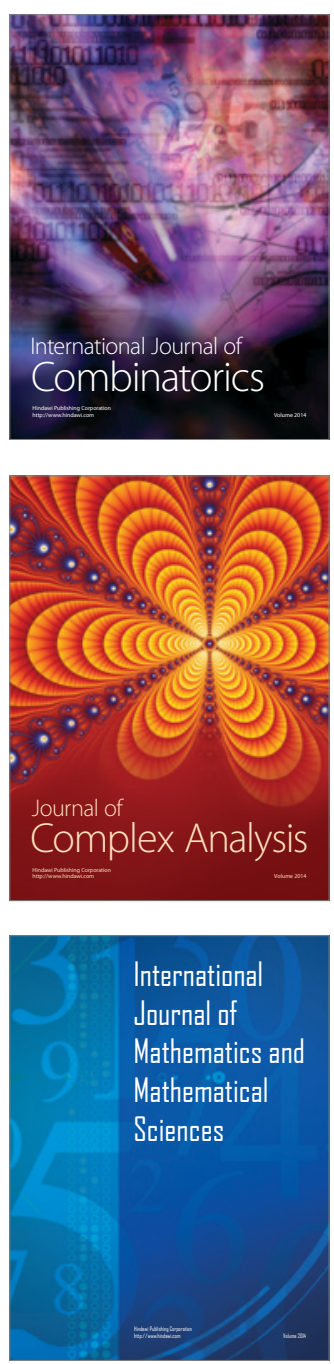
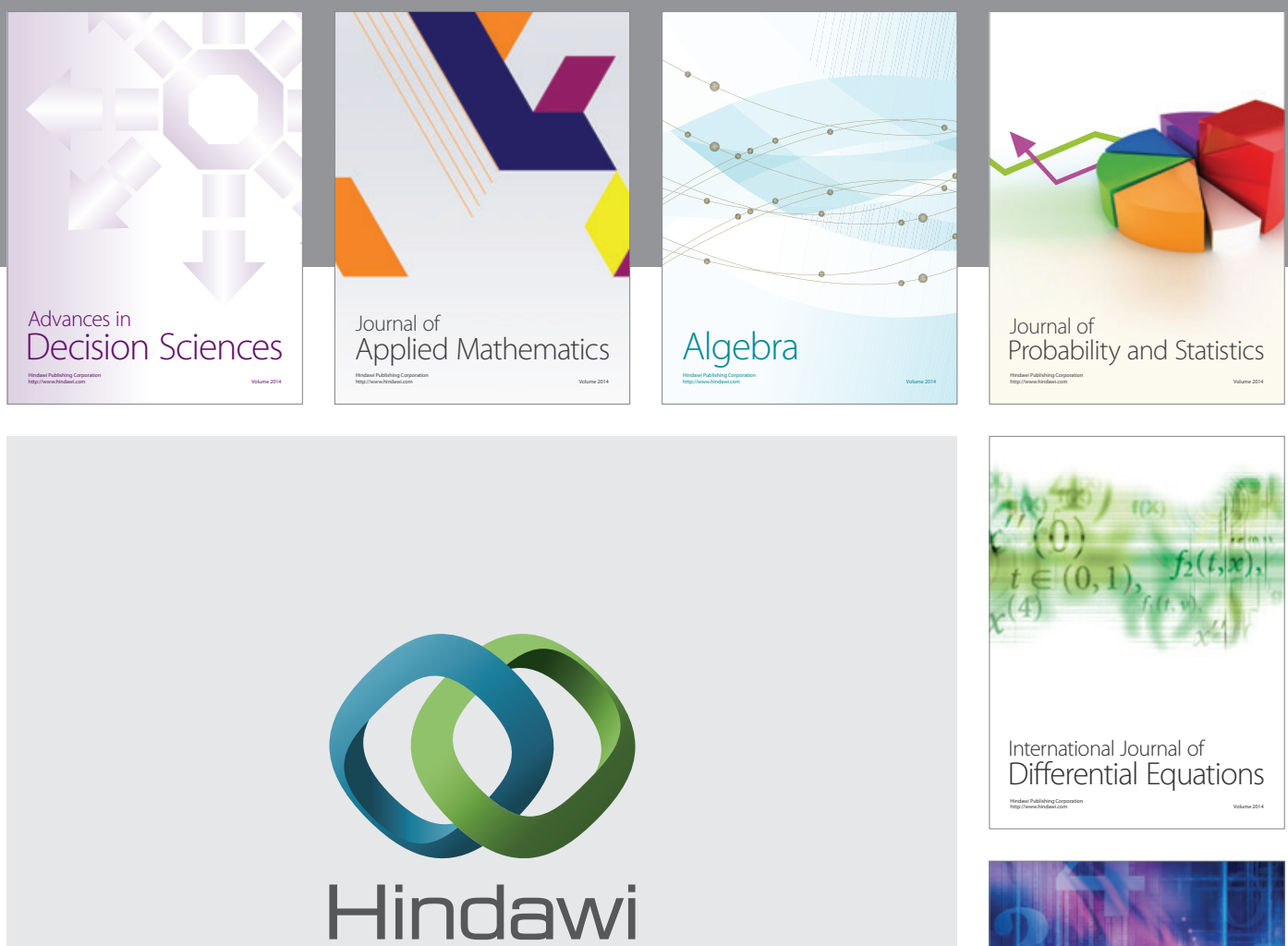

Submit your manuscripts at http://www.hindawi.com
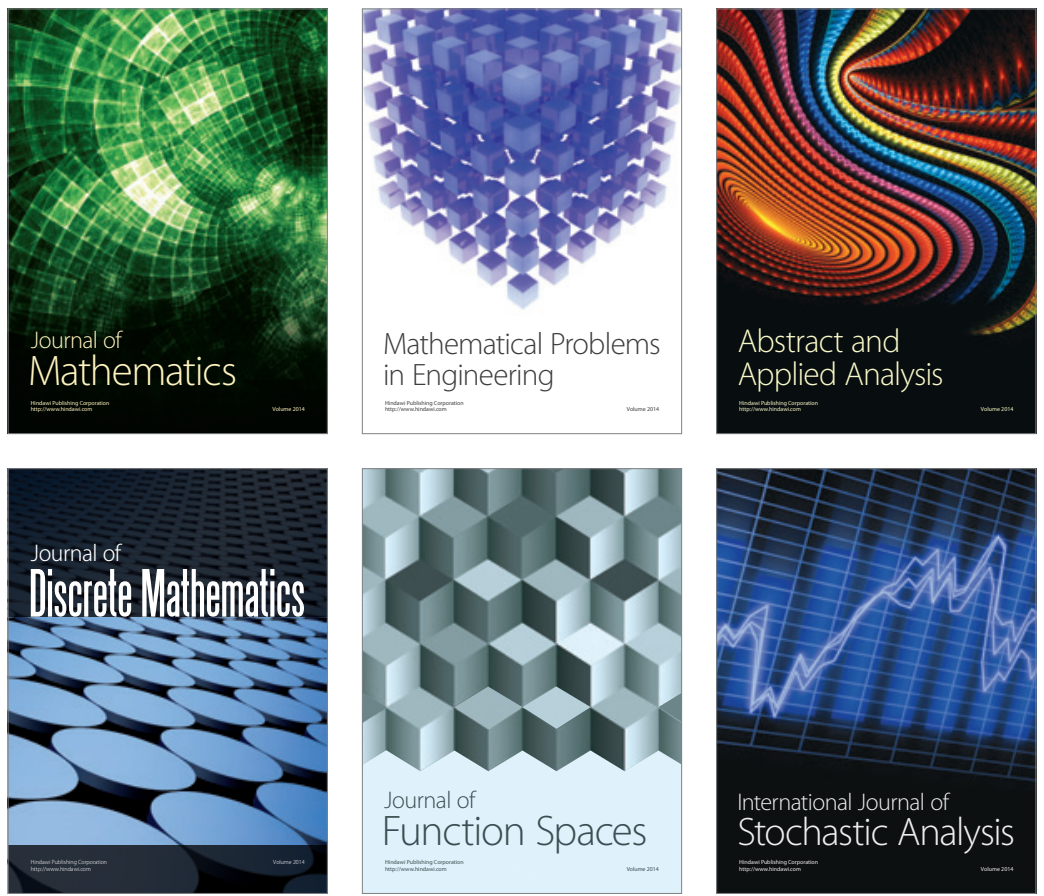

Journal of

Function Spaces

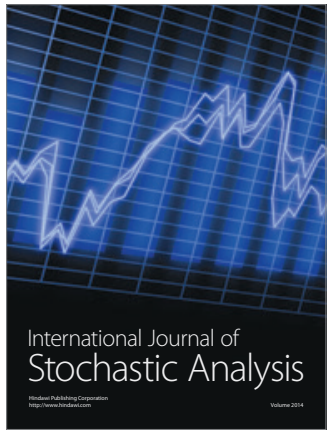

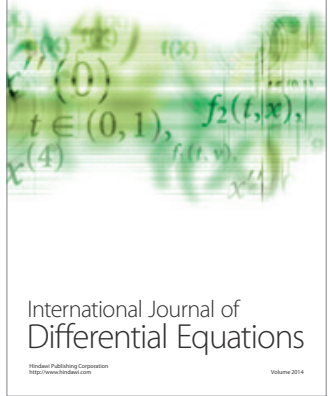
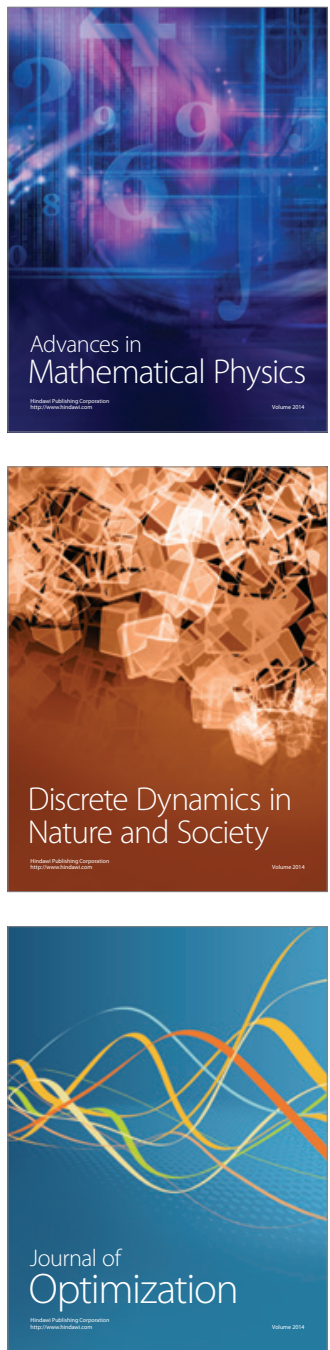